\title{
Anabases
}

ANABASES Traditions et réceptions de l'Antiquité

4 | 2006

Varia

\section{Rééditer Franz Cumont : pourquoi ? comment?}

\section{Corinne Bonnet}

\section{OpenEdition}

Journals

Édition électronique

URL : http://journals.openedition.org/anabases/3014

DOI : 10.4000/anabases.3014

ISSN : 2256-9421

\section{Éditeur}

E.R.A.S.M.E.

\section{Édition imprimée}

Date de publication : 1 octobre 2006

Pagination : 267-270

ISSN : 1774-4296

\section{Référence électronique}

Corinne Bonnet, «Rééditer Franz Cumont: pourquoi ? comment? », Anabases [En ligne], 4 | 2006, mis en ligne le 01 janvier 2012, consulté le 21 octobre 2019. URL : http://journals.openedition.org/ anabases/3014; DOI : 10.4000/anabases.3014 
Anabases 4 (2006), p. $267-270$

\section{Rééditer Franz Cumont : pourquoi ? comment?}

CORINne BONnET

La plupart des gens placent leur ambition dans la famille. Ils espèrent que leurs enfants réaliseront un jour ce qu'ils n'ont pu accomplir et travaillent avant tout pour ceux en qui ils voient comme une prolongation de leur personnalité. Mais il se trouve des hommes - et je crains que nous n'en soyons l'un et l'autre - à qui les circonstances ont refusé cet espoir et ces joies. Pour eux, leur œuvre peut devenir les filles qui feront l'objet de leur complaisance. Elles aussi porteront leur nom et recevront l'empreinte de leur caractère. Elles grandiront suivant les principes qu'on leur aura inculqués, elles s'animeront d'une vie qui dépassera de beaucoup les formes d'une existence humaine et iront elles aussi toujours croissant et se multipliant.

Lettre de F. Cumont à R. Warocqué, 4 août $1904^{1}$

L'ACADEMIA BELGICA de Rome a le privilège de conserver, depuis plus de cinquante ans, les archives de Franz Cumont (1868-1947), l'un des principaux historiens des religions et des sciences du XXe siècle ${ }^{2}$. En collaboration avec l'Institut Historique Belge de Rome, avec pour maittre d'œuvre l'éditeur turinois Nino Aragno, l'Academia Belgica entreprend une réédition des œuvres majeures du savant belge, à cent ans et plus de la publication de ses premières grandes monographies sur Mithra et les "Religions orientales 3 ». Bien des livres de Cumont sont devenus des perles pour bibliophiles, tandis que les bibliothèques institutionnelles disposent rarement d'une

1 Citée par A. VerbanCK, Science et collection. Histoire d'une amitié. La collection d'antiquités classiques de Raoul Warocqué au Musée Royal de Mariemont (Belgique), 1870-1917, in A.-F. LAURENS (éd.), L'anticomanie. La collection d'antiquités aux XVIII et XIXe siècles, Paris, 1992, p. 203.

2 Cf. www.academiabelgica.it

3 F. Cumont, Textes et monuments figurés relatifs aux mystères de Mithra, Bruxelles, 18941899 ; Les mystères de Mithra, Bruxelles, 1900 ; Les religions orientales dans le paganisme romain, Paris, 1906 (4e éd. 1929). 
collection complète de ses œuvres, abondantes et éparpillées dans un certain nombre collections. La constitution d'un " corpus cumontien " - comme il en existe pour d'autres savants marquants du siècle passé, A. Warburg, F. Nietzsche ou M. Weber, pour ne citer que quelques noms $-s^{\prime}$ impose donc comme une entreprise utile et féconde dans la perspective de l'histoire des sciences de l'Antiquité et, au-delà, de l'histoire intellectuelle européenne.

\section{Pourquoi rééditer Cumont?}

On écartera d'emblée, à la base de ce projet, toute perspective " hagiographique " ou célébrative. Certes, cette entreprise, coordonnée par le directeur de l'Academia Belgica, W. Geerts, et par moimême, en collaboration avec un Comité scientifique international, se nourrit de la conscience d'un précieux héritage intellectuel, mais elle se veut résolument critique et scientifique. Parcourir et proposer de nouveau les travaux de Cumont, c'est s'interroger sur les conditions intellectuelles dans lesquelles sa pensée s'est formée et épanouie; c'est aussi mesurer le chemin parcouru de lui à nous, sur le plan des thématiques et des concepts autant que des méthodes. Relire et faire relire Cumont, c'est donc avant tout questionner l'histoire et l'historiographie, la sienne et la nôtre, dans l'optique de l'histoire culturelle des XIXe et XXe siècles.

Les enjeux scientifiques et historiques de l'époque de Cumont ne sont nullement négligeables : l'on peut dire que ses travaux s'inscrivent sur la toile de fond d'une intense fermentation intellectuelle. C'est l'époque où les nationalismes s'affirment et débouchent sur une compétition scientifique, ainsi que sur deux guerres mondiales qui entraînèrent une mobilisation des savants et du discours scientifique au service des armes et des particularismes. C'est aussi l'époque où se constituent les grands corpora, épigraphiques, littéraires, numismatiques, iconographiques, vitrine du " grand atelier ${ }^{4}$ " de l'Altertumswissenschaft. C'est encore l'époque de l'exploration archéologique intensive du monde classique et oriental, de Pompéi à Byblos, en passant par Delphes et Pergame. C'est le temps des grands chantiers pourvoyeurs d'objets destinés aux musées de l'Occident et de nouveaux questionnements quant aux rencontres et aux transferts culturels d'une rive à l'autre de la Méditerranée. L'époque de Cumont, c'est celle où l'histoire des religions se dégage de la théologie et de la philologie, pour devenir une discipline à part entière. C'est le temps tourmenté du modernisme et d'une certaine chasse aux sorcières qui entama jusqu'à la carrière de Cumont lui-même, ami de Loisy. Autant d'ingrédients - mais il en est d'autres - d'une époque occupée à inventer une nouvelle modernité qui questionne la tradition classique, la conteste ou l'exalte. L'interaction profonde entre un auteur et son contexte, entre le sujet historien et son objet d'histoire, fait de l'œuvre de Cumont un carrefour d'idées et d'expériences, un palimpseste de l'histoire ancienne et contemporaine.

Il ne faut pas non plus oublier que, dans certains domaines, en particulier dans l'histoire des religions et dans l'histoire des sciences, les travaux de Cumont ont été fondateurs et restent, à bien des égards, des points de repère essentiels. Sa pensée représente à l'évidence un moment historiographique hautement significatif que le progrès des connaissances et l'évolution des questionnements ont sans cesse sollicité. Qu'on adhère à ses reconstructions ou qu'on les critique, Cumont est une référence obligée de notre savoir historique. Qu'il s'agisse de sa conception des

4 Je renvoie à C. BonneT, Le "grand atelier de la science ". Franz Cumont et l'Altertumswissenschaft. Héritages et émancipations. Des études universitaires à la Première Guerre mondiale (1888-1923), Bruxelles-Rome 2005. 
"religions orientales ", de la genèse du culte de Mithra, de sa lecture symbolique des images, de l'apport des mages chaldéens, du rapport entre religion et astrologie ou encore du culte impérial, sa manière de travailler, d'analyser, de faire de l'histoire ne laisse pas indifférent. C'est pourquoi une réédition d'ensemble s'inscrit encore et toujours dans l'actualité scientifique.

\section{Comment rééditer Cumont?}

Le projet que nous présentons ici prévoit la réédition des œuvres majeures de Franz Cumont, ce qui signifie une bonne vingtaine de monographies, auxquelles on ajoutera divers volumes de Scripta minora (par exemple : des recueils thématiques d'articles) et de Parerga (par exemple : correspondances, carnets de voyage, inédits, etc.). Il s'agit donc d'un projet de longue haleine qui s'étalera sur de nombreuses années et qui prévoit la publication de deux volumes par an, à partir de 2006. Le premier volume de la série, préparé par Françoise Van Haeperen et moi-même, sera Les religions orientales dans le paganisme romain, et paraîtra, cent ans après sa première édition, en novembre 2006, à l'occasion d'un Colloque International sur "Les religions orientales dans le monde grec et romain : cent ans après Cumont ", qui se tiendra à Rome, à l'Academia Belgica. On trouvera ci-dessous la liste de la première tranche de rééditions.

Il ne s'agira pas simplement de reproposer, sic et simpliciter, la " parole du maitre ", mais de la replacer dans une perspective historique et historiographique. Partant, le texte original sera précédé par une introduction substantielle due aux éditeurs scientifiques de chaque volume et suivi par un appendice contenant la transcription des notes manuscrites de Cumont figurant dans ses propres volumes.

Dans l'introduction, on s'efforcera de mettre les travaux de Cumont en perspective, tant en termes de genèse qu'en termes de réception et de postérité. On montrera comment les acquis documentaires plus récents, comment les débats qui ont fait suite à ses publications ont infléchi la lecture défendue par le savant belge. En restituant l'état de la recherche et des connaissances à l'époque de Cumont, en utilisant ses archives pour éclairer l'amont et l'aval de ses livres, on permettra aux lecteurs modernes de mesurer l'originalité, les qualités et les défauts, les limites et la portée, l'impact et l'actualité des textes de Cumont. On appréciera aussi la place qu'il occupe dans les réseaux intellectuels de son époque.

L'appendice, quant à lui, proposera une transcription intégrale des notes que Cumont a prises dans ses exemplaires interfoliés, conservés à l'Academia Belgica. Il s'agit, pour l'essentiel, de compléments documentaires sous la forme de références aux sources ou aux publications, de remarques, de corrections orthographiques ou stylistiques, parfois même de passages de lettres. Ce supplément d'informations, qui constituera une plus-value importante et totalement inédite, représente de fait une trace historiographique à divers niveaux : les notes montrent le fonctionnement d'un chantier ouvert et dynamique au cœur de l'" atelier » historique de Cumont; elles sont révélatrices de sa manière d'interagir avec la communauté scientifique, puisqu'à l'origine de ces notes, on trouve fréquemment des communications ou recensions de collègues, et elles révèlent, enfin, le souci de Cumont de mettre ses analyses à l'épreuve de l'actualité scientifique, souvent dans le but d'en renforcer la validité et l'autorité.

Autour de l'Academia Belgica et de l'héritage scientifique de Franz Cumont, par le biais de cette ambitieuse réédition, mais aussi des recherches portant sur sa correspondance, ses voyages, ses archives, ses photos, est donc en train de se nouer un riche réseau scientifique international à l'image du parcours intellectuel d'un savant qui a su marquer son temps par la profondeur de son 
érudition et la perspicacité de ses analyses, et décloisonner les savoirs au sein des sciences de l'Antiquité. Toutes ces activités visent, en définitive, à préciser le sens et la portée de l'œuvre d'un grand historien qui avait " le courage de travailler le bloc informe et le coup d'œil d'en faire jaillir la lumière ", selon la belle expression que Mommsen ${ }^{5}$ réserva à Cumont.

\section{Programmation de la première tranche de rééditions :}

1. Les religions orientales dans le paganisme romain, 4 e éd., Paris, 1929

Fr. Van Haeperen (Louvain) et C. Bonnet (Toulouse).

2. Lux Perpetua, Paris, 1949

Br. Rochette (Liège) et A. Motte (Liège).

3. Les Mystères de Mithra, Bruxelles, 1900

A. Mastrocinque (Verona) et N. Belayche (Paris, EPHE).

4. Recherches sur le symbolisme funéraire des Romains, Paris, 1942

J. Ch. et J. Balty.

5. Comment la Belgique fut romanisée. Essai historique, Bruxelles, 1914

X. Deru (Lille) et G. Lehman-Delerive (CNRS, Lille).

6. Studia Pontica, 1-3, Bruxelles, 1903-1910

V. Krings (Toulouse) et I. Tassignon (Bruxelles, Bibliothèque royale).

7. Les mages hellénisés. Zoroastre, Ostanès et Hystaspe, d'après la tradition grecque (avec J. Bidez), 2 vol., Paris, 1938

P. Bonnechère (Montréal) et P. Marchetti (Namur-Louvain).

8. L'Égypte des astrologues, Bruxelles, 1937

F. Colin (Strasbourg) et D. Juste (Bruxelles).

9. Afterlife in Roman Paganism, New Haven, 1922

J. Scheid (Collège de France) et Ch. Frateantonio (Collège de France).

10. Études syriennes, Paris, 1917

E. Gubel (Bruxelles, KMKG) et P.L. Gatier (CNRS, Lyon).

11. Fouilles de Doura-Europos, 1922-1923, Paris, 1926

T. Kaizer (Durham) et P. Leriche (CNRS, Paris).

\begin{tabular}{l} 
CORINNE BONNET \\
\hline Université de Toulouse II - Le Mirail \\
UFR d'Histoire, Arts et Archéologie \\
5, allées Antonio Machado \\
F-31058 Toulouse cedex 9 \\
cbonnet@wanadoo.fr
\end{tabular}

$5 \quad$ Lettre de Theodor Mommsen à Paul Frédéric du 22 mai 1910. 